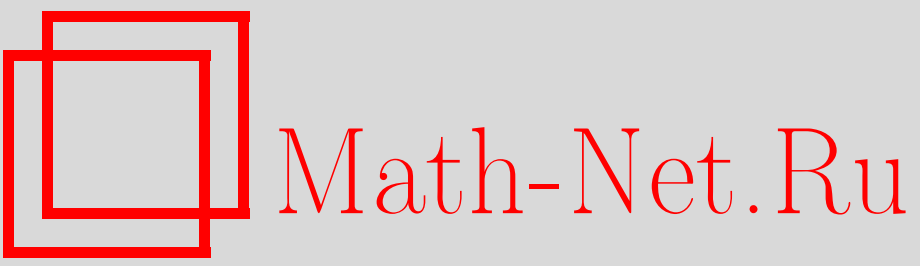

А. Т. Филиппов, Интегрируемые модели $(1+1)$-мерной дилатонной гравитации, взаимодействующей со скалярной материей, ТМФ, 2006, том 146, номер 1, 115131

DOI: https://doi.org/10.4213/tmf2013

Использование Общероссийского математического портала Math-Net.Ru подразумевает, что вы прочитали и согласны с пользовательским соглашением

http://www.mathnet.ru/rus/agreement

Параметры загрузки:

IP : 18.207 .199 .55

26 апреля 2023 г., 13:52:44 
ТЕОРЕТИЧЕСКАЯ

И МАТЕМАТИЧЕСКАЯ

ФИЗИКА

Том 146, № 1

январь, 2006

(C) 2006 г.

А. Т. Филиппов ${ }^{*}$

\title{
ИНТЕГРИРУЕМЫЕ МОДЕЛИ $(1+1)$-МЕРНОЙ ДИЛАТОННОЙ ГРАВИТАЦИИ, ВЗАИМОДЕЙСТВУЮЩЕЙ СО СКАЛЯРНОЙ МАТЕРИЕЙ
}

\begin{abstract}
Достаточно подробно описан класс явно интегрируемых моделей $(1+1)$ мерной дилатонной гравитации, взаимодействующей со скалярными полями. Уравнения движения этих моделей редуцируются к системам уравнений Лиувилля со связями, наложенными на энергию и импульс. В явном виде построено общее решение уравнений и связей в терминах киральных полей (модулей) и кратко обсуждены некоторые обобщения основных интегрируемых моделей. Эти модели могут быть связаны с теориями супергравитации в высших размерностях, но в работе они рассматриваются в основном вне зависимости от такой интерпретации. Приводится также краткий обзор других интегрируемых моделей двумерной дилатонной гравитации.
\end{abstract}

Ключевые слова: дилатонная гравитация, интегрируемые модели, солитон, уравнение Лиувилля.

\section{1. ВВЕДЕНИЕ}

Как хорошо известно, $(1+1)$-мерная дилатонная гравитация, взаимодействующая со скалярными полями материи, является надежной моделью для описания некоторых аспектов черных дыр в высших размерностях, космологических моделей и бран. Связь между высокими и низкими размерностями, установленная в различных контекстах гравитации и теории струн, в ряде случаев позволяет найти общее решение или некоторые специальные классы решений теорий в высших размерностях ${ }^{1)}$. Например, сферически-симметричная гравитация, взаимодействующая с абелевыми калибровочными полями и безмассовыми скалярными полями материи, точно

\footnotetext{
${ }^{1)}$ Более подробное обсуждение этой связи и дальнейшие ссылки см., например, в работах [1], [2], а также в следующем разделе.
}

*Объединенный институт ядерных исследований, Дубна, Московская обл., Россия. E-mail: Alexandre.Filippov@jinr.ru 
редуцируется к $(1+1)$-мерной дилатонной гравитации и может быть явно решена, если скалярные поля суть не зависящие от координат константы. Такие решения могут описывать некоторые интересные физические объекты: сферические статические черные дыры, простые космологические модели и т. д. Однако в случае, когда скалярные поля материи, которые, судя по всему, играют значительную роль в космологии, не постоянны, известно не много точных аналитических решений теорий в высших размерностях. Соответственно двумерные модели дилатонной гравитации, нетривиально взаимодействующей со скалярной материей, обычно не являются интегрируемыми.

Для получения интегрируемых моделей подобного рода следует принять некоторые серьезные аппроксимации, другими словами, деформировать исходную двумерную модель, полученную прямой размерной редукцией реалистических теорий в высших размерностях ${ }^{2)}$. Тем не менее эти модели могут качественно описывать некоторые специальные физически интересные решения теорий гравитации или супергравитации в высших размерностях, связанные с низкоэнергетическим пределом теорий суперструн.

\section{2. НЕКОТОРЫЕ ИНТЕГРИРУЕМЫЕ МОДЕЛИ $(1+1)$-МЕРНОЙ ДИЛАТОННОЙ ГРАВИТАЦИИ, ВЗАИМОДЕЙСТВУЮЩЕЙ СО СКАЛЯРНОЙ МАТЕРИЕЙ}

Эффективный лагранжиан $(1+1)$-мерной дилатонной гравитации, взаимодействующей со скалярными полями $\psi_{n}$, который может быть получен размерной редукцией сферически-симметричной (супер)гравитации в высших размерностях, можно, как правило, (локально) преобразовать к виду ${ }^{3)}$

$$
\mathcal{L}^{(2)}=\sqrt{-g}\left[\varphi R(g)+V(\varphi, \psi)+\sum_{n} Z_{n}(\varphi, \psi) g^{i j} \partial_{i} \psi_{n} \partial_{j} \psi_{n}\right] .
$$

\footnotetext{
2) Заметим, что некоторые важные реализации четырехмерного пространства-времени с симметриями, определяемыми двумя коммутирующими векторами Киллинга, также могут быть описаны двумерной дилатонной гравитацией. Например, цилиндрические гравитационные волны описываются $(1+1)$-мерной дилатонной гравитацией, взаимодействующей с одним скалярным полем. Стационарная аксиально-симметричная чистая гравитация может быть описана $(0+2)$-мерной дилатонной гравитацией, взаимодействующей с одним скалярным полем (здесь возможна связь с указанным цилиндрическим случаем посредством аналитического продолжения пространственных переменных на мнимые значения). Подобные же, но более общие модели дилатонной гравитации были также получены в теории струн. Некоторые из них можно решить с использованием современных математических методов, развитых в теории солитонов.

3) По поводу подробных мотивировок и конкретных примеров см. [1], где также приведены ссылки на другие имеющие отношение к предмету работы. Из-за ограниченного объема статьи здесь приводятся только абсолютно необходимые ссылки.
} 
Здесь $g_{i j}\left(x^{0}, x^{1}\right)-(1+1)$-мерная метрика общего положения с сигнатурой $(-1,1)$, $g \equiv \operatorname{det}\left|g_{i j}\right|, \quad R$ - кривизна Риччи двумерного пространства-времени,

$$
d s^{2}=g_{i j} d x^{i} d x^{j}, \quad i, j=0,1 .
$$

Эффективные потенциалы $V$ и $Z_{n}$ зависят от дилатона $\varphi\left(x^{0}, x^{1}\right)$ и $N-2$ скалярных полей $\psi_{n}\left(x^{0}, x^{1}\right)$ (заметим, что $Z_{n}<0$ ). Они могут зависеть от других параметров, характеризующих "родительскую" теорию в высшей размерности (например, от зарядов, вводимых при решении уравнений для абелевых полей). Мы здесь в основном рассматриваем простой кинетический член, когда $Z_{n}(\varphi, \psi)=Z_{n}(\varphi)$, или даже с постоянным $Z_{n}$, не зависящим от полей. В уравнении (1) мы также использовали преобразование Вейля для исключения градиентного дилатонного члена.

Для упрощения выводов мы используем уравнения движения в метрике светового конуса, $d s^{2}=-4 f(u, v) d u d v$. Варьируя сначала этот лагранжиан в общих координатах и далее переходя к координатам светового конуса, получаем уравнения движения

$$
\begin{gathered}
\partial_{u} \partial_{v} \varphi+f V(\varphi, \psi)=0 \\
f \partial_{i}\left(\frac{\partial_{i} \varphi}{f}\right)=\sum_{n} Z_{n}\left(\partial_{i} \psi_{n}\right)^{2}, \quad i=u, v \\
\partial_{v}\left(Z_{n} \partial_{u} \psi_{n}\right)+\partial_{u}\left(Z_{n} \partial_{v} \psi_{n}\right)+f V_{\psi_{n}}(\varphi, \psi)=\sum_{m} Z_{m, \psi_{n}} \partial_{u} \psi_{m} \partial_{v} \psi_{m}, \\
\partial_{u} \partial_{v} \ln |f|+f V_{\varphi}(\varphi, \psi)=\sum_{n} Z_{n, \varphi} \partial_{u} \psi_{n} \partial_{v} \psi_{n}
\end{gathered}
$$

где $V_{\varphi}=\partial_{\varphi} V, \quad V_{\psi_{n}}=\partial_{\psi_{n}} V, \quad Z_{n, \varphi}=\partial_{\varphi} Z_{n}$ и $Z_{m, \psi_{n}}=\partial_{\psi_{n}} Z_{m}$. Эти уравнения не независимы, в действительности (6) следует из (3)-(5). Альтернативным образом,

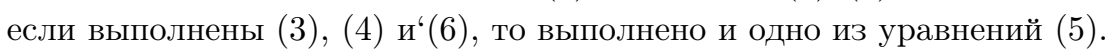

Рассмотрим решение с постоянным скалярным полем $\psi \equiv \psi_{0}$ (“скалярный вакуум"). Оно существует, если $V_{\psi}\left(\varphi, \psi_{0}\right)=0$ (см. уравнение (5)). Связи (4) можно теперь решить, поскольку их правые части тождественно равны нулю. Несложно доказать, что существуют такие киральные поля $a(u)$ и $b(v)$, что $\varphi(u, v) \equiv \varphi(\tau)$ и $f(u, v) \equiv \varphi^{\prime}(\tau) a^{\prime}(u) b^{\prime}(v)$, где $\tau \equiv a(u)+b(v)$ (штрихи обозначают производные по соответствующим аргументам). Используя этот результат, легко показать, что уравнение (3) имеет интеграл $\varphi^{\prime}+N(\varphi)=M$, где $N(\varphi)$ определяется уравнением $N^{\prime}(\varphi)=V\left(\varphi, \psi_{0}\right)$, а $M-$ константа (интеграл) движения. Горизонт, определяемый как нуль метрики $h(\tau) \equiv M-N(\varphi)$, существует, потому что уравнение $M=N(\varphi)$ имеет по крайней мере одно решение в некотором интервале значений $M$. Эти решения являются в действительности одномерными (“автоматически” размерно редуцированными) и могут интерпретироваться как черные дыры (Шварцшильда, Райсснера-Нордстрёма и др.) или как космологические модели. На самом деле скалярный вакуум теории эквивалентен чистой дилатонной гравитации ${ }^{4)}$, которая яв-

\footnotetext{
${ }^{4)}$ Если $V=V(\varphi)$ и $Z_{n} \equiv 0$, то теория (1) называется чистой дилатонной гравитацией. Она интегрируема при произвольном потенциале $V(\varphi)$.
} 
ляется топологической теорией, что и объясняет произошедшее радикальное упрощение уравнений движения. Эти факты известны уже в течение значительного времени и были выведены многими авторами с использованием различных подходов (см. библиографию, например, в недавнем обзоре [2]).

Свойства общих дилатонных теорий гравитации (1) существенно сложнее. Эти теории не редуцируются к одномерным моделям и, вообще говоря, не являются интегрируемыми. Если $Z_{n} \neq 0$, то теорию можно проинтегрировать только при очень специальных потенциалах $V(\varphi, \psi)$ и $Z_{n}(\varphi, \psi)$. Грубо говоря, двумерные модели можно проинтегрировать, если потенциалы $Z_{n}$ преобразуются к постоянным или если потенциал $V$ нулевой. В первом случае $V$ должен иметь очень специальный вид, описываемый ниже. Во втором случае, для того чтобы теория была явно аналитически интегрируема ${ }^{5)}$, потенциалы $Z_{n}$ должны выражаться весьма специальными функциями.

При поиске интегрируемых $(1+1)$-мерных моделей может оказаться полезным рассмотрение также "смешанных" случаев. Грубо говоря, это означает следующее. Предположим, что имеются три различных типа скалярных полей материи $-\psi_{n}, \chi_{k}$ и $\sigma_{s}$. Характеристические свойства этих полей таковы: потенциал $V$ зависит от $\varphi$ и $\psi_{n} ; Z_{n}$ и $Z_{k}$ постоянны, например, $Z_{n}=Z_{k}=-1 ; Z_{s}=Z_{s}(\varphi)$ не зависят от $\sigma$ и $\psi$. Таким образом, $Z$-часть лагранжиана (1) имеет вид

$$
\sqrt{-g} g^{i j}\left[\sum_{n} Z_{n} \partial_{i} \psi_{n} \partial_{j} \psi_{n}+\sum_{k} Z_{k} \partial_{i} \chi_{k} \partial_{j} \chi_{k}+\sum_{s} Z_{s}(\varphi) \partial_{i} \sigma_{s} \partial_{j} \sigma_{s}\right] .
$$

При взгляде на уравнения (3)-(6) видно, что $\chi_{k}-$ свободные безмассовые поля (т.е. $\left.\partial_{u} \partial_{v} \chi_{k}=0\right)$, уравнения $(3)$ и $(5)$ для $\varphi$ и $\psi_{n}$ не зависят от остальных полей и, наконец, уравнения для $\sigma_{s}$ линейны. Правые части связей (4),

$$
\sum_{n} Z_{n}\left(\partial_{i} \psi_{n}\right)^{2}+\sum_{k} Z_{k}\left(\partial_{i} \chi_{k}\right)^{2}+\sum_{s} Z_{s}(\varphi)\left(\partial_{i} \sigma_{s}\right)^{2}, \quad i=u, v,
$$

зависят от всех трех типов полей материи, поэтому поля не расцепляются (что естественно, поскольку некоторые линейные комбинации связей дают полную энергию и импульс системы, на которые наложено условие обращения в нуль). Наиболее общую систему такого типа можно явно проинтегрировать, если ее нелинейный сектор явно интегрируется, а связи можно явно решить при произвольных решениях для полей $\sigma$ и $\chi$. K сожалению, в столь общей задаче связи решить невозможно. Как показано в следующем разделе, когда имеются только поля $f, \varphi$ и $\psi$ (при постоянных полях $\sigma$ ), каждая связь включает только функции одной переменной (соответственно $u$ или $v$ ). Однако в обсуждаемых здесь интегрируемых моделях это условие не удовлетворяется для члена $Z_{s}(\varphi)\left(\partial_{i} \sigma_{s}\right)^{2}$. По этой причине мы рассмотрим главным

\footnotetext{
5) Мы называем теорию явно интегрируемой, если можно явно записать ее общее решение аналитически в терминах достаточного числа произвольных функций, в нашем случае - киральных свободных полей и их производных. Для дальнейшего использования в физических задачах нам требуются достаточно простые выражения для общего решения.
} 
образом модели с полями материи $\psi$ и $\chi$. Тем не менее полезно не забывать про $\sigma$ поля по двум причинам. Во-первых, в ряде случаев эти слагаемые можно учесть как возмущения. Вторая причина состоит в следующем. При дальнейших редукциях к размерностям $(0+1)$ или $(1+0)$ поля $\sigma$ могут допускать точное решение, давая дополнительные слагаемые в потенциал $V$. Получающаяся в результате теория может быть интегрируемой или приближенно интегрируемой. Мы не рассматриваем здесь такие возможности и в дальнейшем сконцентрируемся на теориях с полями $\psi$ и $\chi$.

Но сначала обсудим второй “чистый” случай (включение $\chi$-полей тривиально, и мы его опустим). Итак, предположим, что $V \equiv 0, \psi \equiv \chi \equiv 0$ и $Z_{s}=Z(\varphi)$. Тогда из первого уравнения имеем $\varphi=u+v \equiv r$ (с точностью до преобразования координат $u \mapsto a(u), \quad b \mapsto b(v))$, а скалярные поля $\sigma$ удовлетворяют линейному уравнению $(t \equiv u-v)$

$$
\left(\partial_{r}^{2}-\partial_{t}^{2}\right) \sigma(r, t)+\frac{Z^{\prime}(r)}{Z(r)} \partial_{r} \sigma(r, t)=0
$$

Если $Z(\varphi)=-\varphi$, это уравнение Эйлера-Дарбу, для общего решения которого можно записать достаточно сложное интегральное представление (см., например, [3]). Более того, выражение для метрики очень сложно проанализировать. В общем случае это не позволяет найти явное аналитическое выражение для метрики. Таким образом, такие теории можно, наверное, называть интегрируемыми, но я бы сказал, что они не являются явно интегрируемыми. Однако если $Z_{n}(\varphi)$ - решения одномерного уравнения Лиувилля, то потенциалы, возникающие из-за такого $Z_{n}(r)$, являются “безотражательными," уравнения для $\sigma$ явно интегрируются и решения даются простыми функциями киральных свободных безмассовых полей $a(u)$ и $b(v)$ (см. [4]). При этом метрика явно выражается через эти киральные поля, и, таким образом, теория явно (и элементарно) интегрируема. Эти решения могут описывать плоские волны скалярной материи, взаимодействующей с гравитацией, или некоторые неоднородные космологические модели.

Качественное различие между решениями уравнения (9) для “безотражательных" и "реалистичных" (соответствующих $Z=-\varphi$ ) потенциалов становится более ясным, если использовать следующие изящные решения уравнения (9) при $Z=-\varphi^{2 \lambda+1}$, $\lambda>-1 / 2$. Первое решение было по существу найдено Пуассоном:

$$
\sigma(r, t)=\int_{0}^{\pi} d \alpha q(t-r \cos \alpha) \sin ^{2 \lambda} \alpha
$$

а второе было получено позднее Хобсоном ${ }^{6)}$ :

$$
\sigma(r, t)=\int_{0}^{\infty} d \alpha q(t \pm r \operatorname{ch} \alpha) \operatorname{sh}^{2 \lambda} \alpha
$$

где $q(x)$ - произвольная "производящая функция."

\footnotetext{
6) Известные еще с XIX века, эти решения и их обобщения можно найти в переиздании по-прежнему полезной старой книги [5].
} 
Потенциал, соответствующий $Z=-\varphi^{2 \lambda+1}$, становится "безотражательным," если $\lambda=l+1 / 2$ с целочисленным $l$. Действительно, тогда уравнение (9) эквивалентно

$$
\left(\partial_{r}^{2}-\partial_{t}^{2}\right) \bar{\sigma}(r, t)-\frac{l(l+1)}{r^{2}} \bar{\sigma}(r, t)=0
$$

где $\bar{\sigma} \equiv r^{l+1} \sigma$. Потенциал $l(l+1) / r^{2}$ является специальным случаем "безотражательных" потенциалов, рассмотренных в работе [4], при которых решение этого волнового уравнения можно явно записать в виде линейной комбинации свободного безмассового поля $\chi=a(t+r)+b(t-r)$ и его производных (с зависящими от $r$ коэффициентами). Легко доказать, что решения (10) и (11) редуцируются к таким локальным полям, если $\lambda=l+1 / 2$ с целочисленным $l$. Если $l$ не является целочисленным, решение представляет собой непрерывную суперпозицию волн с различными "скоростями" $\sec \alpha$ или $\mathrm{ch}^{-1} \alpha$. В этом смысле решение является, вообще говоря, нелокальным и в действительности не более простым, чем то, которое использовалось в работе [3]. Поучительно переписать уравнение (10) с полуцелым $\lambda$ в виде

$$
\sigma(r, t)=\frac{1}{r} \int_{t-r}^{t+r} d x q(x)[(x-(t-r))((t+r)-x)]^{l}
$$

Если $l=0$, то находим

$$
\sigma(r, t)=\frac{1}{r}[Q(t+r)-Q(t-r)]
$$

где $Q^{\prime}(x) \equiv q(x)$. Аналогично можно найти локальные выражения для $\sigma$ в терминах безмассового свободного поля для любого целочисленного $l$.

Исходно решения (10) и (11) применялись для описания простых цилиндрических волн (см., например, [5]). Позднее было показано, что их можно обобщить для описания достаточно сложных цилиндрических гравитационных волн (см., например, работу [6], в которой также обсуждается обобщение этого классического решения в контексте твисторной программы Пенроуза). Действительно, цилиндрические гравитационные волны простейшего вида, выведенные Эйнштейном и Розеном [7], можно найти, решая именно уравнение (9). Для нахождения цилиндрических гравитационных волн наиболее общего вида требуется решить нелинейное уравнение

$$
\partial_{t}\left(r M^{-1} \partial_{t} M\right)-\partial_{r}\left(r M^{-1} \partial_{r} M\right)=0
$$

где $M$ - симметричная $(2 \times 2)$-матрица, зависящая от цилиндрической метрики. Это уравнение можно решить путем обобщения формулы Пуассона [6] или с помощью более общих методов, разработанных в теории солитонов.

В действительности уравнение (14), описывающее цилиндрические гравитационные волны, является специальным случаем полевых уравнений для $\sigma$-моделей, взаимодействующих с гравитацией. Это уравнение и его обобщения можно попытаться решить с помощью методов обратной задачи рассеяния (ОЗР) теории солитонов. 
Применение этих методов берет начало с работ [8], в которых рассматривалась интегрируемость стационарной аксиальной гравитации. Дальнейшие результаты и ссылки на многочисленные публикации на эту тему можно найти в работах [9]. Методы решения интегрируемых двумерных теорий, сводящиеся к решению уравнений, аналогичных уравнению (14), не элементарны и по этой причине не включены в нашу классификацию материальных членов (15), которые могут приводить к элементарно решаемой двумерной дилатонной гравитации. В наших обозначениях для моделей, которые могут быть проинтегрированы с помощью ОЗР, имеем $V=0, Z_{n}=Z_{k}=0$, но, вообще говоря, более сложную структуру $\sigma$-членов:

$$
\sum_{\alpha, \beta} Z_{\alpha \beta}(\varphi ; \sigma) \partial_{i} \sigma_{\alpha} \partial_{j} \sigma_{\beta}
$$

В простых случаях можно преобразовать эти члены к диагональному виду, аналогичному выражению (7), но с потенциалами $Z$, зависящими от $\sigma$-полей. Например, лагранжиан двумерной дилатонной гравитации, полученный в работе [10] (см. формулу (2.8)) для стационарной аксиально-симметричной эйнштейновской гравитации, легко преобразовать к виду, соответствующему нашей “общей” дилатонной гравитации (1), однако это не помогает решить уравнения движения элементарными методами. С другой стороны, мы увидим, что существует довольно широкий класс интересных с физической точки зрения интегрируемых двумерных теорий с простым (постоянным!) потенциалом $Z_{n}$, но с достаточно сложными потенциалами $V$ [1], [11], [12].

Наиболее изученными примерами таких интегрируемых моделей (принадлежащих к моделям первого "чистого" типа с постоянным $Z_{n}$ ) являются модель Каллана-Гиддингса-Харви-Строминджера $(\mathrm{K} \Gamma \mathrm{XC})\left(V=g_{0}\right)$ и модель ДжакиваТейтельбойма (ДжТ) $\left(V=g_{1} \varphi\right)$. В КГХС-модели $R=0$, а в ДжТ-модели $R=-g_{1}$. Существенное обобщение этих моделей, в котором двумерная кривизна не является конформно-постоянной $\left(V=g_{+} e^{g \varphi}+g_{-} e^{-g \varphi}\right)$, была предложена нами в работе [11]. Подобно КГХС- и ДжТ-моделям, эта модель принадлежит к "смешанному" типу, в котором имеются свободные поля $\chi_{k}$. Она была названа моделью 2-Лиувилля, поскольку поля $\psi_{ \pm} \equiv \ln f \pm \varphi$ удовлетворяют двум уравнениям Лиувилля. Уравнения Лиувилля, конечно, явно решаются в терминах двух пар киральных свободных безмассовых полей $a_{ \pm}(u), b_{ \pm}(v)$. Используя связи (4), можно выразить одно из свободных скалярных полей $\chi$ через $a, b$ и другие $\chi$-поля. Таким образом, можно было бы подумать, что модель 2-Лиувилля явно решена в работе [11]. Однако осталась одна "небольшая" проблема: правые части связей являются отрицательно определенными, тогда как левые части могут менять знак. В работе [11] нам не удалось найти явные аналитические выражения для киральных полей $a$ и $b$, которые полностью удовлетворяют связям. Явное решение связей (4) было построено только пять лет спустя, когда мы решили гораздо более общую модель $N$-Лиувилля [1], [12]. Здесь мы покажем, как можно постороить такой класс моделей, и представим его полное решение в терминах киральных полей модулей. 


\section{3. (1+1)-МЕРНАЯ МОДЕЛЬ $N$-ЛИУВИЛЛЯ И ЕЕ РЕШЕНИЕ}

Пусть теория определяется лагранжианом

$$
\mathcal{L}^{(2)}=\sqrt{-g}\left[\varphi R(g)+V(\varphi, \psi)+g^{i j}\left(\sum_{n} Z_{n} \partial_{i} \psi_{n} \partial_{j} \psi_{n}+\sum_{k} Z_{k} \partial_{i} \chi_{k} \partial_{j} \chi_{k}\right)\right]
$$

с потенциалами

$$
|f| V=\sum_{n=1}^{N} 2 g_{n} e^{q_{n}}, \quad Z_{n}=Z_{k}=-1 .
$$

Здесь $f$ - метрика светового конуса, $d s^{2}=-4 f(u, v) d u d v$ и

$$
q_{n} \equiv F+a_{n} \varphi+\sum_{m=3}^{N} \psi_{m} a_{m n} \equiv \sum_{m=1}^{N} \psi_{m} a_{m n}
$$

где $\psi_{1}+\psi_{2} \equiv \ln |f| \equiv F \quad\left(f \equiv \varepsilon e^{F}, \varepsilon= \pm 1\right), \quad \psi_{1}-\psi_{2} \equiv \varphi$, откуда $a_{1 n}=1+a_{n}$, $a_{2 n}=1-a_{n}$. Варьируя лагранжиан (16) по $N-2$ скалярным полям, дилатону и $g_{i j}$, а затем переходя к метрике на световом конусе, находим $N$ уравнений движения для $N$ функций $\psi_{n}$ :

$$
\epsilon_{n} \partial_{u} \partial_{v} \psi_{n}=\sum_{m=1}^{N} \varepsilon g_{m} e^{q_{m}} a_{m n}, \quad \epsilon_{1}=-1, \quad \epsilon_{n}=+1 \quad \text { при } \quad n \geqslant 2,
$$

и для двух связей:

$$
C_{i} \equiv f \partial_{i}\left(\frac{\partial_{i} \varphi}{f}\right)+\sum_{n=3}^{N}\left(\partial_{i} \psi_{n}\right)^{2}=4 D_{i}(i), \quad i=(u, v),
$$

где $4 D_{i}(i) \equiv-\sum\left(\partial_{i} \chi_{k}\right)^{2}$ - вклад свободных полей $\chi$ (см. уравнение $\left.(8)\right)$, которые зависят от одной переменной, поскольку $\chi_{k}=\alpha_{k}(u)+\beta_{k}(v)$ (здесь и далее $k=$ $1, \ldots, K)$.

При произвольных коэффициентах $a_{m n}$ эти уравнения движения не интегрируемы. Однако, как было предложено в работе [12], уравнения (19) интегрируемы, а связи (20) можно решить, если $N$-компонентные векторы $v_{n} \equiv\left(a_{m n}\right)$ псевдоортогональны. Тогда уравнения (19) редуцируются к $N$ независимым явно интегрируемым уравнениям Лиувилля для $q_{n}$ :

$$
\partial_{u} \partial_{v} q_{n}-\tilde{g}_{n} e^{q_{n}}=0
$$

где $\tilde{g}_{n}=\varepsilon \lambda_{n} g_{n}, \quad \lambda_{n}=\sum \epsilon_{m} a_{m n}^{2}$ и $\varepsilon \equiv f /|f|$ (заметим, что уравнения для $q_{n}$ зависят от $\epsilon_{n}$ и $a_{m n}$ только неявно, через нормирочный множитель $\left.\lambda_{n}\right)^{7)}$.

\footnotetext{
7) Здесь мы предполагаем, что $\lambda_{n} \neq 0$ и $g_{n} \neq 0$. В противном случае решение для связей должно быть преобразовано довольно очевидным образом. Мы также используем обозначение $\gamma_{n} \equiv \lambda_{n}{ }^{-1}$.
} 
Выражение для исходного поля в терминах поля Лиувилля $q_{n}$ можно найти, используя соотношения ортогональности для $a_{m n}(m \neq n)$ и определение $\lambda_{n} \equiv \gamma_{n}^{-1}$ (при $m=n$ ), объединенные в равенства

$$
\sum_{l=1}^{N} \epsilon_{l} a_{l m} a_{l n}=\lambda_{n} \delta_{m n} \equiv \gamma_{n}^{-1} \delta_{m n}
$$

Их можно записать в матричном виде, если определить матрицы $a=\left(a_{m n}\right), \gamma=$ $\left(\gamma_{m} \delta_{m n}\right)$ и $\epsilon=\left(\epsilon_{m} \delta_{m n}\right)$ :

$$
a^{\mathrm{T}} \epsilon a=\gamma^{-1}, \quad a \gamma a^{\mathrm{T}}=\epsilon .
$$

Важно отметить, что, кроме этих соотношений, матрица $а$ удовлетворяет условиям $a_{1 n}=1+a_{n}, a_{2 n}=1-a_{n}$.

Теперь, используя (22) или (23), можно обратить определение (18) и получить соотношения

$$
\psi_{m}=\epsilon_{m} \sum_{n=1}^{N} a_{m n} \gamma_{n} q_{n}, \quad F=-2 \sum_{n=1}^{N} \gamma_{n} a_{n} q_{n}, \quad \varphi=-2 \sum_{n=1}^{N} \gamma_{n} q_{n} .
$$

Выписывая первое соотношение для $n=1,2$, выражая $a_{1 n}$ и $a_{2 n}$ через $a_{n}$ и используя линейную независимость функций $\psi_{n}$, можно найти чрезвычайно полезные тождества ("правила сумм") для $\gamma_{n}, a_{n}$, и $a_{m n}$ при $m \geqslant 3$. Имеем

$$
\begin{aligned}
& \psi_{1} \equiv-\psi_{1} \sum_{n=1}^{N} \gamma_{n}\left(1+a_{n}\right)^{2}-\psi_{2} \sum_{n=1}^{N} \gamma_{n}\left(1-a_{n}^{2}\right)-\sum_{m \geqslant 3}^{N} \psi_{m} \sum_{n=1}^{N} a_{m n}\left(1+a_{n}\right) \gamma_{n} \\
& \psi_{2} \equiv \psi_{1} \sum_{n=1}^{N} \gamma_{n}\left(1-a_{n}^{2}\right)+\psi_{2} \sum_{n=1}^{N} \gamma_{n}\left(1-a_{n}\right)^{2}+\sum_{m \geqslant 3}^{N} \psi_{m} \sum_{n=1}^{N} a_{m n}\left(1-a_{n}\right) \gamma_{n} .
\end{aligned}
$$

Таким образом, мы немедленно находим, что должны удовлетворяться следующие тождества:

$$
\begin{aligned}
& \sum_{n=1}^{N} \gamma_{n}=0, \quad \sum_{n=1}^{N} \gamma_{n} a_{n}=-\frac{1}{2}, \quad \sum_{n=1}^{N} \gamma_{n} a_{n}^{2}=0, \\
& \sum_{n=1}^{N} a_{m n} \gamma_{n}=\sum_{n=1}^{N} a_{m n} a_{n} \gamma_{n}=0, \quad m \geqslant 3 .
\end{aligned}
$$

Кроме того, используя соотношения ортогональности (22), можно доказать, что одна и только одна норма $\gamma_{n}$ отрицательна. Выберем, таким образом, $\gamma_{1}<0$, а другие $\gamma_{n}$ положительными. В физически мотивированных моделях параметры $a_{m n}$, $\gamma_{n}$ и $g_{n}$ могут удовлетворять некоторым дополнительным соотношениям. Например, знаки $\gamma_{n}$ и $g_{n}$ могут быть скоррелированы так, что $g_{n} / \gamma_{n}<0$. Однако такие соотношения не следуют из условий ортогональности, и мы их не рассматриваем ${ }^{8)}$.

\footnotetext{
${ }^{8)}$ На самом деле константы взаимодействия никак не связаны с интегрируемостью и могут быть произвольными числами.
} 
Наиболее важным является тот факт, что связи могут быть решены явно. Сначала запишем решения уравнений Лиувилля (21) в виде, который следует из свойств конформной симметрии уравнений Лиувилля [13]:

$$
e^{-q_{n} / 2}=a_{n}(u) b_{n}(v)-\frac{1}{2} \tilde{g}_{n} \bar{a}_{n}(u) \bar{b}_{n}(v) \equiv X_{n}(u, v)
$$

где киральные поля $a_{n}(u), b_{n}(v), \bar{a}_{n}(u)$ и $\bar{b}_{n}(v)$ удовлетворяют уравнениям (не путать $a_{n}(u)$ с $a_{n}$, которые использовались выше)

$$
a_{n}(u) \bar{a}_{n}^{\prime}(u)-a_{n}^{\prime}(u) \bar{a}_{n}(u)=1, \quad b_{n}(v) \bar{b}_{n}^{\prime}(v)-b_{n}^{\prime}(v) \bar{b}_{n}(v)=1 .
$$

Используя уравнения (29), можно выразить $\bar{a}$ и $\bar{b}$ через $a$ и $b$ и записать $X_{n}$ в виде

$$
X_{n}(u, v)=a_{n}(u) b_{n}(v)\left[1-\frac{1}{2} \tilde{g}_{n} \int \frac{d u}{a_{n}^{2}(u)} \int \frac{d u}{b_{n}^{2}(v)}\right] .
$$

С помощью несложных преобразований связи (20) можно переписать в виде

$$
C_{u} \equiv 4 \sum_{n=1}^{N} \gamma_{n} \frac{a_{n}{ }^{\prime \prime}(u)}{a_{n}(u)}=4 D_{u}(u), \quad C_{v} \equiv 4 \sum_{n=1}^{N} \gamma_{n} \frac{b_{n}{ }^{\prime \prime}(v)}{b_{n}(v)}=4 D_{v}(v)
$$

В первую очередь заметим, что из уравнений (18) и (23) легко найти тождества

$$
-\partial_{i} F \partial_{i} \varphi+\sum_{n=3}^{N}\left(\partial_{i} \psi_{n}\right)^{2}=\sum_{n=1}^{N} \gamma_{n}\left(\partial_{i} q_{n}\right)^{2}
$$

Отсюда следует, что

$$
C_{i}=\sum_{n=1}^{N} \gamma_{n}\left(\left(\partial_{i} q_{n}\right)^{2}-2 \partial_{i}^{2} q_{n}\right)=4 \sum_{n=1}^{N} \gamma_{n} \frac{\partial_{i}^{2} X_{n}}{X_{n}}
$$

Теперь из определения $X_{n}$ нетрудно видеть, что

$$
\frac{\partial_{u}^{2} X_{n}}{X_{n}}=\frac{a_{n}^{\prime \prime}(u)}{a_{n}(u)}, \quad \frac{\partial_{v}^{2} X_{n}}{X_{n}}=\frac{b_{n}^{\prime \prime}(v)}{b_{n}(v)} .
$$

Используя соотношение $\sum \gamma_{n}=0$, эти связи можно решить явно. Покажем это для $C_{u}$; вывод для $C_{v}$ аналогичен. Введем временно обозначение $a_{n}^{\prime}(u) / a_{n}(u) \equiv$ $r_{n}(u)$, используем его в выражении для $C_{u}$ и сдвинем все $r_{n}$ на некоторую неизвестную вспомогательную функцию $R(u)$, т.е. определим $\rho_{n}(u) \equiv r_{n}(u)+R(u)$. Таким образом, можно выразить $C_{u}$ через $\rho_{n}(u)$ и $R(u)$ :

$$
\frac{1}{4} C_{u}=\sum_{n=1}^{N} \gamma_{n} \frac{a_{n}^{\prime \prime}(u)}{a_{n}(u)}=\sum_{n=1}^{N} \gamma_{n}\left(r_{n}^{\prime}+r_{n}^{2}\right)=\sum_{n=1}^{N} \gamma_{n}\left[\rho_{n}^{\prime}(u)+\rho_{n}^{2}(u)-2 \rho_{n}(u) R(u)\right]
$$

где мы использовали тождество $\sum \gamma_{n}=0$ (здесь и далее пределы суммирования опущены, когда суммирование проводится по всем возможным значениям $n=1, \ldots, N$ 
и $k=1, \ldots, K)$. Теперь легко видеть, что связь $C_{u}=4 D_{u}$ будет решена, если мы возьмем

$$
R(u)=\frac{1}{2}\left[\sum_{n=1}^{N} \gamma_{n}\left(\rho_{n}^{\prime}(u)+\rho_{n}^{2}(u)\right)-D_{u}(u)\right]\left[\sum_{n=1}^{N} \gamma_{n} \rho_{n}(u)\right]^{-1},
$$

где $\rho_{n}(u)$ - произвольные функции. Таким образом, если выбрать $a_{n}^{\prime}(u) / a_{n}(u)=$ $\rho_{n}-R(u)$, где $R$ дается уравнением (36), то связь $C_{u}=4 D_{u}$ будет удовлетворяться ${ }^{9)}$.

Введем теперь новые поля модулей

$$
\mu_{m}(u) \equiv \rho_{m}(u)-\frac{1}{2}\left[\sum_{n=1}^{N} \gamma_{n} \rho_{n}^{2}(u)-D_{u}(u)\right]\left[\sum_{n=1}^{N} \gamma_{n} \rho_{n}(u)\right]^{-1} .
$$

Используя определение и тождество $\sum \gamma_{n}=0$, легко проверить, что

$$
\sum \gamma_{n} \mu_{n}(u)=\sum \gamma_{n} \rho_{n}(u), \quad \sum \gamma_{n} \mu_{n}^{2}(u)=D_{u}(u)=-\frac{1}{4} \sum\left(\alpha_{k}^{\prime}(u)\right)^{2} .
$$

Повторив этот вывод для второй связи $C_{v}$ и определив $\nu_{n}(v)-v$-аналог модулей $\mu_{n}(u)$, окончательно запишем обе связи в терминах произвольных киральных полей $\mu_{n}(u), \nu_{n}(v), \alpha_{k}(u), \beta_{k}(v)$ :

$$
\sum \gamma_{n} \mu_{n}^{2}(u)+\frac{1}{4} \sum\left(\alpha_{k}^{\prime}(u)\right)^{2}=0, \quad \sum \gamma_{n} \nu_{n}^{2}(v)+\frac{1}{4} \sum\left(\beta_{k}^{\prime}(v)\right)^{2}=0 .
$$

Эти связи эквивалентны исходным (20), если поля $f, \varphi, \psi, \chi$ являются решениями уравнений движения.

Возвращаясь к исходным полям модулей $\rho_{n}$ и их выражениям через $a_{n}^{\prime}(u) / a_{n}(u) \equiv$ $r_{n}(u)$ (и к их $v$-аналогам), можно видеть, что связи эквивалентны дифференциальным уравнениям первого порядка для $a_{n}(u)$ и $b_{n}(v)$ :

$$
\frac{a_{n}^{\prime}(u)}{a_{n}(u)}=\mu_{n}(u)-\frac{1}{2} \frac{\sum \gamma_{n} \mu_{n}{ }^{\prime}(u)}{\sum \gamma_{n} \mu_{n}(u)}, \quad \frac{b_{n}^{\prime}(v)}{b_{n}(v)}=\nu_{n}(v)-\frac{1}{2} \frac{\sum \gamma_{n} \nu_{n}{ }^{\prime}(v)}{\sum \gamma_{n} \nu_{n}(v)}
$$

где $\mu_{n}(u)$ и $\nu_{n}(v)$ - произвольные функции, удовлетворяющие связям (39). Это небольшое "чудо" позволяет явно решить уравнения движения (включая связи) в терминах "достаточного" числа произвольных функций. Число независимых произвольных киральных функций в правой части уравнения (40) равно $2(N-1+K)$, где $N-2$ - число скалярных материальных полей $\psi$, а $K$ - число свободных скалярных материальных полей $\chi$. Вместе с “гравитационными” степенями свободы $\varphi, F$ (или $\left.\psi_{1}, \psi_{2}\right)$ у теории без связей было бы $2(N+K)$ киральных степеней свободы. Мы сейчас покажем, что остаточные координатные преобразования позволяют еще уменьшить число независимых произвольных киральных функций до $2(N-2+K)$.

\footnotetext{
9) Заметим, что $\rho_{n}-R$ не являются независимыми функциями. В действительности киральные поля $a_{n}$ зависят от $N-1$ произвольных независимых функций переменной $u$. Это станет очень скоро ясно из несколько иного представления для решения связи.
} 
Интегрируя дифференциальные уравнения первого порядка (40) для $a_{n}(u)$ и $b_{n}(v)$, найдем общее решение $N$-лиувиллевской дилатонной гравитации в терминах киральных полей модулей $\mu_{n}(u)$ и $\nu_{n}(v)$, удовлетворяющих связям (39):

$$
\begin{aligned}
& a_{n}(u)=\left|\sum \gamma_{m} \mu_{m}\right|^{-1 / 2} \exp \left(\int d u \mu_{n}(u)\right) \\
& b_{n}(v)=\left|\sum \gamma_{m} \nu_{m}\right|^{-1 / 2} \exp \left(\int d v \nu_{n}(v)\right) .
\end{aligned}
$$

Выберем произвольные киральные $\chi$-поля $\alpha_{k}(u)$ и $\beta_{k}(v)$. Тогда поля модулей $\mu_{n}(u)$ и $\nu_{n}(v)$ не будут независимыми из-за связей (39). Кроме того, можно использовать остаточные координатные преобразования $u \rightarrow U(u)$ и $v \rightarrow V(v)$ для того, чтобы выбрать два калибровочных (координатных) условия. Покажем, что запись

$$
\left|\sum \gamma_{n} \mu_{n}(u)\right| \equiv U^{\prime}(u), \quad\left|\sum \gamma_{n} \nu_{n}(v)\right| \equiv V^{\prime}(v)
$$

действительно эквивалентна выбору $(U, V)$ в качестве новой системы координат. Для этого определим новые киральные поля

$$
A_{n} \equiv \exp \left(\int d u \mu_{n}(u)\right), \quad B_{n} \equiv \exp \left(\int d v \nu_{n}(v)\right),
$$

которые мы будем рассматривать как функции новых координат $(U, V)$ (в силу неявных преобразований (42)). Используя соотношения (41) и (42), можно переписать уравнение (30) в терминах $A_{n}(U), B_{n}(V)$ :

$$
Y_{n}(U, V)=A_{n}(U) B_{n}(V)\left[1-\frac{1}{2} \tilde{g}_{n} \int \frac{d U}{A_{n}^{2}(U)} \int \frac{d V}{B_{n}^{2}(V)}\right],
$$

где мы определили

$$
Y_{n}(U, V) \equiv\left[U^{\prime}(u) V^{\prime}(v)\right]^{1 / 2} X_{n}(u, v) .
$$

Теперь, используя введенные выше определения, можно найти метрику $f$ :

$$
f(u, v)=U^{\prime}(u) V^{\prime}(v) \prod_{n=1}^{N}\left[Y_{n}(U, V)\right]^{4 \gamma_{n} a_{n}} \equiv \bar{f}(U, V) U^{\prime}(u) V^{\prime}(v)
$$

дилатон $\varphi$ и скалярные поля $\psi_{m}(m \geqslant 3)$ :

$$
e^{\varphi}=\prod_{n=1}^{N}\left[Y_{n}(U, V)\right]^{4 \gamma_{n}}, \quad e^{\psi_{m}}=\prod_{n=1}^{N}\left[Y_{n}(U, V)\right]^{-2 a_{m n} \gamma_{n}} .
$$

Мы видим, что $d s^{2}=-4 f(u, v) d u d v \equiv-4 \bar{f}(U, V) d U d V$ и, таким образом, все выражается в терминах новых координат $(U, V)$.

Связи (39) для параметров модулей легко решить, выражая один из модулей через другие. Однако может оказаться удобнее и поучительнее ввести новые модули, 
которые проясняют топологическую природу пространства модулей и могут поэтому допускать интересную топологическую классификацию решений $N$-лиувиллевской теории. Для упрощения обозначений определим новые модули для случая нулевых $\chi$-полей (общий случай не имеет существенных отличий). Полагая $\chi \equiv 0$, введем следующие единичные $(N-1)$-векторы (напомним, что $\gamma_{1}<0$ и $\gamma_{k}>0$ при $\left.k \geqslant 2\right)$ :

$$
\hat{\xi}_{k}(u) \equiv \frac{\mu_{k}(u) \sqrt{\gamma_{k}}}{\mu_{1}(u) \sqrt{\left|\gamma_{1}\right|}}, \quad \hat{\eta}_{k}(v) \equiv \frac{\nu_{k}(v) \sqrt{\gamma_{k}}}{\nu_{1}(v) \sqrt{\left|\gamma_{1}\right|}}, \quad k=2, \ldots, N
$$

Эти векторы, движущиеся по $(N-2)$-мерной единичной сфере $S^{(N-2)}$, определяют решение с точностью до выбора системы координат, которую можно фиксировать с помощью приведенных выше калибровочных условий (42). Они теперь выглядят следующим образом:

$$
\begin{array}{cc}
U^{\prime}(u)=\left|\gamma_{1} \mu_{1}(u)\right|\left(1-\cos \theta_{\xi}(u)\right), & V^{\prime}(v)=\left|\gamma_{1} \nu_{1}(v)\right|\left(1-\cos \theta_{\eta}(v)\right), \\
\cos \theta_{\xi}(u)=\sum_{k=2}^{N} \hat{\gamma}_{k} \hat{\xi}_{k}(u) \equiv \hat{\gamma} \hat{\xi}, \quad \cos \theta_{\eta}(v)=\sum_{k=2}^{N} \hat{\gamma}_{k} \hat{\eta}_{k}(v) \equiv \hat{\gamma} \hat{\eta},
\end{array}
$$

где $\hat{\gamma}$ - постоянный единичный $(N-1)$-вектор, $\hat{\gamma}_{k}=\left(\gamma_{k} /\left|\gamma_{1}\right|\right)^{1 / 2}$.

Поля модулей $\hat{\xi}(u)$ и $\hat{\eta}(u)$ очень полезны, поскольку они дают простое представление наиболее важных решений и, в частности, делают соотношения между решениями для различных размерностей наглядными. Например, если векторы $\hat{\xi}(u)$ и $\hat{\eta}(v)$ являются постоянными и равными $(\hat{\xi}=\hat{\eta})$, они дают статическое или космологическое решение. Статическое решение имеет горизонт, если $\hat{\xi}=\hat{\eta}=\hat{\gamma}$ (см. [1]). Другое интересное статическое решение, являющееся плоским на бесконечности, дается постоянными векторами, удовлетворяющими условиям $\hat{\xi}=\hat{\eta}=\hat{\delta}$, где $\delta_{k} \equiv\left(\gamma_{k} a_{k} /\left(\left|\gamma_{1}\right| a_{1}\right)\right)^{1 / 2}$ (несложно проверить, что этот вектор действительно имеет единичную длину). Двумерные решения могут быть представлены парами кривых на $S^{(N-2)}$, которые мы обозначим как $(\hat{\xi}(u), \hat{\eta}(v))$. Те решения, которые представляют собой асимптотическую интерполяцию между описанными выше одномерными решениями, имеют особое значение и определяют дополнительную структуру на $S^{(N-2)}$, которую можно использовать для физического обоснования классификации решений $N$-лиувиллевской теории.

Особенно интересными являются новые решения, которые, судя по всему, могут быть локализованы в пространстве (солитоноподобные волны скалярной материи). Они соответствуют постоянным, но не равным векторам $\hat{\xi}$ и $\hat{\eta}$ (это, очевидно, возможно при $N \geqslant 3)$. В координатах $(U, V)(U+V \equiv r, U-V \equiv t)$ решения можно записать как

$$
Y_{n}(U, V)=C_{n} \operatorname{ch}\left[R_{n}^{-1}\left(\left(r-r_{n}\right)-v_{n} t\right)\right],
$$

где параметры $C_{n}, R_{n}, r_{n}, v_{n}$ рационально зависят от модулей и других параметров, характеризующих лагранжиан и систему координат (напомним, что эти параметры не являются независимыми вследствие связей и условий калибровки). Хотя экспоненты $e^{q}=Y_{n}^{-2}$ локализованы в пространстве, этого не обязательно достаточно для 
того, чтобы материальные поля были локализованы. Однако, используя произвол в выборе модулей и других параметров, можно записать истинно локализованное решение. Детальный вывод и исследование этих интересных решений будут представлены в отдельной работе.

Таким образом, мы получили общее решение $(1+1)$-мерной дилатонной гравитаци, взаимодействующей с любым числом скалярных полей. Оно явно выражается в терминах достаточного числа произвольных киральных полей ${ }^{10)}$ и, таким образом, мы можем решить задачу Коши и исследовать решения, описывающие космологическую эволюцию или черные дыры и т.д. Представление общего решения через киральные поля $a_{n}(u)$ и $b_{n}(v)$ может послужить хорошей отправной точкой для попыток квантования нашей $N$-лиувиллевской дилатонной гравитации. Еще более полезными могут быть киральные поля модулей $\mu_{n}(u)$ и $\nu_{n}(v)$ (или $\hat{\xi}_{k}(u)$ и $\hat{\eta}_{k}(v)$ ). В терминах этих полей модулей размерная редукция решений становится весьма наглядной, что может упростить вывод и физическую интерпретацию эволюции одномерных решений и привести к новым подходам к квантованию, основанным на аналогии с простым одномерным случаем ${ }^{11)}$.

\section{4. ОБСУЖДЕНИЕ И ПЕРСПЕКТИВЫ}

Представленные в настоящей работе аналитически явно интегрируемые модели могут быть интересны с точки зрения различных применений. Наиболее очевидным образом их можно использовать для построения первых приближений к неинтегрируемым, вообще говоря, теориям. Реалистические теории, описывающие черные дыры и космологические модели, обычно не являются интегрируемыми. Однако явные общие решения интегрируемых аппроксимаций могут позволить построить различные варианты теории возмущений.

Например, сферически-симметричные статические черные дыры, не минимально взаимодействующие со скалярными полями, описываются интегрируемой $(0+1)$ мерной $N$-лиувиллевской моделью. Подобным же образом сферически-симметричные космологические модели могут описываться $(1+0)$-мерной $N$-лиувиллевской теорией. Однако соответствующая $(1+1)$-мерная теория не является интегриру-

\footnotetext{
${ }^{10)}$ На первый взгляд, выражения для полей $\bar{f}, \varphi, \psi_{m}$ выглядят нелокальными. Однако, обозначая неопределенные интегралы в выражении (44) новыми киральными полями, мы видим, что $Y_{n}$ являются по существу локальными функциями новых полей и их производных.

11) Одномерные модели, получаемые с помощью простой размерной редукции, легко квантовать. Наша формулировка близка к одномерной теории настолько, насколько это возможно, и это поддерживает надежду найти наконец квантовую версию $N$ лиувиллевской теории. Следует подчеркнуть, что до настоящего времени интересные результаты, полученные в квантовой теории Лиувилля, пролили не много света на $N$ лиувиллевскую теорию. Это можно объяснить тем фактом, что основная трудность и основное содержание $N$-лиувиллевской теории, в отличие от стандартной теории Лиувилля, заключаются в связях.
} 
емой, поскольку потенциалы скалярного взаимодействия $Z_{n}$ непостоянны (в действительности $Z_{n} \sim \varphi$ ). Для получения приближенных аналитических решений $(1+1)$-мерной теории можно попытаться аппроксимировать $Z_{n}$ с помощью правильно выбранных констант.

Этот подход можно комбинировать с недавно предложенной аналитической теорией возмущений, позволяющей находить решения близко к горизонту для наиболее общих неинтегрируемых $(0+1)$-мерных дилатонных гравитационных теорий [14]. Вблизи горизонта можно использовать интегрируемую $(1+1)$-мерную дилатонную гравитацию (при $Z_{n}=-1$ ) как хорошую аппроксимацию реалистической теории (при $Z_{n}$, зависящем от дилатона $\varphi$ ).

С точки зрения применения в космологии большой интерес представляет поведение $(1+1)$-мерных решений вблизи сингулярности при $\varphi=0$ (см., например, [15]). Интегрируемые $(1+1)$-мерные $N$-лиувиллевские теории вблизи сингулярности могут давать в лучшем случае грубую качественную аппроксимацию точных решений. Количественная аппроксимация может быть получена, если сначала асимптотически решить точную теорию в окрестности $\varphi=0$, а затем сшить асимптотические решения с решениями интегрируемой теории. Для реализации такой программы требуются очень простые и явные аналитические решения интегрируемой теории. Наша простая модель, имеющая решения, представленные в терминах модулей $\hat{\xi}$ и $\hat{\eta}$, может служить хорошей исходной точкой для такой деятельности. Конечно, прежде чем применения к реалистическим космологическим моделям станут возможными, необходимо подробно исследовать и полностью классифицировать и интерпретировать поведение $(1+1)$-мерных решений и их точную связь с $(1+0)$-мерной редукцией.

Редукция из размерности $1+1$ как к размерности $1+0$ ("космологическая"), так и к размерности $0+1$ ("статическая" или "черная дыра") является особенно наглядной в представлении модулей для решений $(1+1)$-мерной $N$-лиувиллевской модели. Однако, как подчеркивалось в работе [1], вся процедура размерной редукции должна быть заново рассмотрена с более общей точки зрения, с учетом более общих размерных редукций. Детальному рассмотрению обобщенных размерных редукций будет посвящена отдельная работа.

ПРИЛОЖЕНИЕ

Дадим схематическое описание простого подхода к решению псевдоортогональных условиий для $a_{m n}$ с дополнительными ограничениями $a_{1 n}=1+a_{n}, a_{2 n}=1-a_{n}$. Уравнения, которые нужно решить, имеют вид

$$
a_{i}+a_{j}=\frac{1}{2} \sum_{n=3}^{N} a_{n i} a_{n j}, \quad i<j .
$$

Эти $N(N-1) / 2$ уравнений нелинейны, однако существует простой рекуррентный алгоритм, сводящий их решение к решению линейных уравнений. Для того чтобы найти его, нужно выбрать, какие из параметров следует рассматривать как неизвестные. Анализируя случаи $N=3$ и $N=4$, можно прийти к следующему удобному

5 Теоретическая и математическая физика, т. 146, № 1, 2006 г. 
выбору деления параметров на неизвестные и произвольным образом фиксированные. Зафиксируем $a_{33}$ и $a_{m n}$ при $m>n, m \geqslant 3$; таким образом, все другие параметры окажутся неизвестными. Сначала рассмотрим уравнения при $i, j=1,2,3$. Обозначая правую часть уравнения (П.1) как $A_{k}$, где $(i j k)=(123)_{\text {циклич}}$, найдем

$$
2 a_{i}=-A_{i}+A_{j}+A_{k}, \quad(i j k)=(123)_{\text {циклич }}
$$

На следующем шаге рассмотрим уравнения (П.1) при $i=1,2,3$ и $j=4$. Они составляют три линейных уравнения для трех неизвестных $a_{4}, a_{34}, a_{44}$, имеющих единственное решение при условии, что правая часть и определитель $\Delta$ не обращаются в нуль.

Теперь должна быть ясна общая конструкция. На $j$-м шаге (при $j=4, \ldots, N)$ мы будем иметь $j-1$ уравнений для $j-1$ неизвестных, $a_{j}$ и $a_{n j}$ при $3 \leqslant n \leqslant j$ :

$$
-2 a_{j}+\sum_{n=3}^{j} a_{n i} a_{n j}=2 a_{i}-\sum_{n=j+1}^{N} a_{n i} a_{n j}, \quad i=1, \ldots, j-1 .
$$

Правые части этих уравнений зависят как от произвольных, так и от ранее найденных параметров. Нетривиальной частью процедуры является то, что, начиная со значения $j=5$, определитель зависит от ранее найденных параметров, и, таким образом, условие $\Delta \neq 0$ сложно контролировать. При $j=4$ определитель

$$
\Delta=2\left[\left(a_{33}-a_{31}\right)\left(a_{43}-a_{42}\right)-\left(a_{33}-a_{32}\right)\left(a_{43}-a_{41}\right)\right]
$$

зависит только от произвольных параметров.

Окончательно запишем параметры для общей модели для $N=3$ :

$$
4 a_{i}=a_{3 i} a_{3 j}-a_{3 j} a_{3 k}+a_{3 k} a_{3 i}, \quad \gamma_{i}^{-1}=\left(a_{3 i}-a_{3 j}\right)\left(a_{3 i}-a_{3 k}\right), \quad(i j k)=(123)_{\text {циклич }} .
$$

Благодарности. При подготовке и написании этой работы я часто вспоминал стимулирующие беседы с М. Савельевым о теории Лиувилля и других интегрируемых теориях. Эти беседы сильно повлияли на мой интерес и подход к предметам, обсуждаемым выше.

Автор благодарит за финансовую поддержку Факультет теоретической физики Университета Турина и INFN (секция Турина), Теоретический отдел ЦЕРНа, МахPlanck-Institut für Physik (Werner-Heisenberg-Institut) в Мюнхене, где были получены некоторые результаты. Автор выражает благодарность П. Фрэ, Д. Люсту, Д. Мэзону и Г. Венециано за полезные обсуждения, а также Г. А. Алексееву за полезные замечания, касающиеся раздела 2 и ссылок. Автор особенно благодарен В. де Альфаро за его поддержку в течение многих лет и весьма плодотворное сотрудничество; в частности, некоторые результаты, представленные в настоящей работе, были получены в тесном сотрудничестве с ним. Эта работа была завершена, когда автор находился в Теоретическом отделе ЦЕРНа; автор высоко ценит теплое гостеприимство и поддержку его сотрудников. Работа выполнена при частичной финансовой поддержке РФФИ (грант № 03-01-0071-а). 


\section{Список литературы}

[1] $\quad$. de Alfaro, A. T. Filippov. Integrable low dimensional models for black holes and cosmologies from high dimensional theories. hep-th/0504101.

[2] D. Grumiller, W. Kummer, D. Vassilevich. Phys. Rep. 2002. V. 369. P. 327; hepth/0204253.

[3] P. Szekeres. J. Math. Phys. 1972. V. 13. P. 286.

[4] А. Т. Филипnов, В. Г. Филипnов. ЯФ. 1998. Т. 61. № 10. С. 1757; hep-th/9803059.

[5] H. Bateman. The Mathematical Analysis of Electrical and Optical Wave-Motion on the Basis of Maxwell's Equations. N.Y.: Dover, 1955.

[6] N. M. J. Woodhouse. Class. Q. Grav. 1989. V. 6. P. 933.

[7] N. Rosen. Phys. Z. der Sowjetunion. 1937. V. 12. P. 366.

[8] В. А. Белинский, В. Е. Захаров. ЖЭТФ. 1978. Т. 75. № 6. С. 1955; D. Maison. Phys. Rev. Lett. 1978. V. 41. P. 521.

[9] H. Nicolai, D. Korotkin, H. Samtleben. Integrable classical and quantum gravity. hepth/9612065; G. Alekseev. Monodromy data parametrization of the spaces of local solutions of integrable reductions of Einstein's field equations. gr-qc/0503043.

[10] P. Breitenlohner, D. Maison. Ann. Inst. H. Poincaré. 1987. V. 46. P. 215.

[11] A. T. Filippov. Mod. Phys. Lett. A. 1996. V. 11. P. 1691; Int. J. Mod. Phys. A. 1997. V. 12. P. 13; hep-th $/ 9605008$.

[12] A. T. Filippov. Phys. Part. Nucl. 2001. V. 32S1. P. 38; ЯФ. 2002. Т. 65. C. 1-5.

[13] J. L. Gervais. Int. J. Mod. Phys. A. 1991. V. 6. P. 2805.

[14] A. T. Filippov, D. Maison. Class. Q. Grav. 2003. V. 20. P. 1779; gr-qc/0210081.

[15] M. Gasperini, G. Veneziano. Phys. Rep. 2003. V. 373. P. 1; hep-th $/ 0207130$. 